\title{
El acaparamiento de tierras de China desde la ecología-mundo: la búsqueda de soja, arroz y caña de azúcar baratos y los límites a la acumulación de capital
}

\author{
Sol Yamila Mora*
}

\section{RESUMEN}

La oleada de inversiones en tierras desatada por la escalada en el precio de los alimentos durante el siglo XXI fue paralela a la prioridad que la República Popular China asignó al control de tierras a fin de responder a la demanda de alimentos de su población, acentuada por su consolidación como un polo de acumulación global. Este trabajo adopta el enfoque de ecología-mundo de Jason Moore para indagar las implicancias del acaparamiento de tierras de China con finalidad alimentaria alrededor del mundo sobre el proceso de acumulación capitalista. Para ello, estudia sus inversiones en tierras en el exterior para la producción de soja, arroz y caña de azúcar, en el período 2000-2019, en base a información extraída de la base de datos Land Matrix.

Se argumenta que el acaparamiento de tierras de China expresa e intensifica las contradicciones en el proceso de acumulación. Por un lado, supone una expansión de la participación del agronegocio chino en el sistema alimentario financiarizado para apropiarse de las tierras indispensables para responder a las necesidades alimentarias de sus trabajadores y su clase media. No obstante, por el otro, los altos costos que la crisis ambiental impone a esos proyectos no solo limitan su ejecución, sino también las posibilidades de una nueva expansión del capitalismo.

El primer apartado caracteriza el acaparamiento de tierras a partir de las nociones de comida barata y hegemonía del capital financiero. Además, introduce el concepto de valor negativo. Tras ello, se desarrollan las razones de las inversiones en tierras de China en función de su posición como polo de acumulación global. La tercera sección se concentra en el acaparamiento de tierras de China para la producción de soja, arroz y caña de azúcar. Finalmente, se reflexiona sobre los límites ambientales a la acumulación de capital mediante el acaparamiento de tierras.

\section{Palabras clave}

Acaparamiento de tierras; China; acumulación de capital; límites ambientales; ecología-mundo.

\section{Tithe}

China's land grabbing from world-ecology: the quest for cheap soybean, rice and sugarcane and the limits to capital accumulation

\section{Extended Abstract}

The surge in land investments unleashed by the escalating food prices during the 21 st century coincided with the priority that the People's Republic of China assigned to the control of lands in other territories in order to respond to its food demand, accentuated by its consolidation as a pole of global accumulation. While the literature questioned the real magnitude of China's participation in the process and explored the causes that led these initiatives to failure, the significance of China's land grab within the dynamics of capitalist accumulation has not been studied enough. Similarly, how the limits that emerge as a result of the environmental crisis affect the implementation of these projects, and with it, the possibilities of accumulation, has not been explored either.

This paper investigates the implications of China's land grabbing for food purposes around the world on the process of capitalist accumulation. With that aim, it studies China's overseas land investments for the production of soybeans, rice and sugarcane in the period 2000-2019. Drawing on Jason Moore's world-ecology approach, it is argued that China's land

DOI:

https://doi.org//0.15366/relacionesinternacionales202I.46.007

Formato de citación recomendado:

MORA, Sol Yamila (202I). "El acaparamiento de tierras de China desde la ecología-mundo: la búsqueda de soja, arroz y caña de azúcar baratos y los límites a la acumulación de capital”, Relaciones Internacionales, n 46, pp. II9138.

* SolYamila MORA Becaria posdoctoral del Consejo Nacional de Investigaciones Científicas y Técnicas (CONICET). Doctora en Ciencias Sociales (FLACSO-Argentina). Investigadora del Área de Estudios Internacionales de la Universidad Nacional de San Martín (EPyGUNSAM). Contacto: smora@unsam. edu.ar

Recibido: 16/09/2020

Aceptado: 20/I 2/2020 
grabbing expresses and intensifies the contradictions in the process of accumulation. On the one hand, it expands the participation of Chinese agribusiness in the financialized food system in order to appropriate the lands essential to meet the food needs of its workers and its middle class. However, on the other hand, the high costs that the environmental crisis imposes on these projects not only limit their implementation, but also the possibilities of a new expansion of capitalism.

In terms of methodology, due to data limitations, the evolution of China's land investments is analyzed through information provided by Land Matrix database. Since this paper focuses exclusively on initiatives linked to the production of soybean, rice and sugarcane for food purposes - the three crops that were concentrated in the land deals in terms of hectares involvedit examines $6 \mathrm{I}$ land deals around the world.Additionally, press information and academic publications are used to discuss certain cases in more detail.

The paper characterizes land grabbing as a response to two current features of the capitalist world-ecology: the end of cheap food and the hegemony of finance capital. The variety of interests around land resulting from both dynamics unleashes a competition to open up the remaining frontiers in the world through land grabbing to appropriate the cheap food that could renew accumulation. Additionally, it introduces the notion of negative value to explore how this outcome can be restricted by the emergence of limits rooted in the environmental crisis that obstructs the restoration of cheap food.

In this context, China's involvement in land grabbing is understood as a manifestation of the dynamics inherent to the development of capitalism after its consolidation as a pole of global accumulation. In order to open up new avenues of accumulation, China promoted the expansion of its agribusiness corporations and the search for investment opportunities in land around the world through the Going Out strategy. This encouraged the opening of frontiers in other territories that would allow China to access cheap food to overcome the biophysical limitations and high costs of domestic agricultural production as well as to reduce imports. However, due to China's distrust of highly concentrated and financialized agricultural markets, another key determinant of these projects was the interest of Chinese agribusiness in increasing its power in the food system to compete with the large grain traders. Both elements explain why rice, soybeans and sugarcane have concentrated Chinese land investments. These crops are not only crucial to China's food security, to the point that it has become one of the largest consumers and importers in the world, but also in that the latter two are highly coveted by finance.

The investments examined show that Latin America and Eastern Russia became the frontier par excellence for soybean production as these regions registered the highest number of agreements linked to this crop in the world. In contrast, the production of rice and sugar cane concentrated in Africa and Asia.Access to these crops is inseparable from the maintenance of China's global leadership in pork and poultry production, as well as the opportunities that the diffusion of hybrid rice and access to cheap sugar cane would provide for seed companies and the processing industry respectively. This demonstrates the unity between accumulation, power and nature that defines world-ecology.

However, this paper found that a significant number of these projects never came to be implemented. This failure can be attributed to the high costs of the initiatives, which hinder their original purpose: the access to cheap food. This is an expression of the negative value that emerges from land grabbing due to the environmental crisis. The location of projects on the remaining external frontiers forces Chinese companies to internalize the environmental degradation present in those areas. The need to convert land that is degraded or unsuitable for agricultural production requires an intensification of the application of increasingly toxic and destructive technologies and inputs, which dramatically raises the costs of the projects. More seriously, sometimes even the use of these techniques is not enough to control the resistance of nature to capital in the form of climatic events such as droughts and floods, epidemics, or the unsuitability of soils for hybrid crops.

It is worth noting that not only does environmental degradation represent a constraint to land grabbing, but that, conversely, land grabbing accentuates the degradation of nature, which casts serious doubts on the effectiveness of this strategy for access to cheap food. In brief, the environmental devastation inherent to land grabbing exacerbates the contradictions of capitalism in a context where the absence of new frontiers hinders the possibilities of accumulation. The conclusions suggest that future research should incorporate other actors and crops, including non-food crops, to develop a better understanding of the effectiveness of land grabbing in restoring cheap nature. In addition, it is necessary to explore the new frontiers that may result from the failure of China's land grab. Finally, more rigorous study is needed on how land grabbing activates negative value.

\section{KEYWORDS}

Land grabbing; China; capital accumulation; environmental limits; world-ecology. 


\section{I}

\section{ntroducción}

La escalada en el precio internacional de los alimentos durante el siglo XXI impulsó una oleada de inversiones en tierras por parte de entidades financieras, inversionistas privados $y$ empresas transnacionales. Este fenómeno fue simultáneo a la prioridad que adquirió para ciertos estados el control de tierras agrícolas en otros territorios a fin de asegurar su abastecimiento alimentario. Ambas tendencias explican la intensidad y las dimensiones que, en el contexto de la crisis alimentaria de 2008, adquirió el acaparamiento global de tierras (Borras et al., 2012; Cotula, 20I2). Aunque esas operaciones ocurren mayoritariamente mediante acuerdos ligados a inversiones, representan un nuevo cercamiento que conlleva la desposesión de bienes comunes, como el agua y los bosques, y su privatización para el establecimiento de la agricultura industrial a gran escala (White et al., 20I2).

De modo paralelo, la reducción de la disponibilidad de tierras agrícolas y agua exacerbó el desafío que representa para la República Popular China garantizar la extraordinaria demanda de alimentos de su población sin interrumpir el ritmo de crecimiento económico (Thomas, 2013) que la consolidó como un polo de acumulación global. Esto desató una expansión de los capitales chinos, facilitada por la estrategia de Go Out para promover la internacionalización de las empresas, en la búsqueda de oportunidades de inversión agrícola alrededor del globo. La faceta más visible de este proceso son las operaciones encabezadas por la Corporación Nacional China de Cereales, Aceites y Comestibles (COFCO) y la Corporación Nacional Química de China (Chemchina)' para proveer a China canales más seguros de abastecimiento de alimentos en el exterior e incrementar su poder en los mercados agrícolas (J. Zhang, 2019). No obstante, el control de tierras en otros estados para la producción de alimentos también fue un objetivo central de esos flujos. Ello convirtió a esta potencia en uno de los mayores inversores en tierras del mundo, con proyectos que se extienden por todos los continentes y a la producción de múltiples cultivos (Chen et al., 2017).

Cabe destacar que a causa de la brecha entre los acuerdos anunciados y los efectivamente ejecutados, la reducida extensión de muchas de las operaciones y el déficit de información exacta de las transacciones, diversas investigaciones cuestionaron la magnitud real de la participación de China en el proceso (Brautigam, 20I5; Hofman y Ho, 20I2; Myers y Jie, 2016; Oliveira, 20I7; $Z$ ha, Xhang y He, 20l8). Entre las razones del fracaso de esas iniciativas se señalaron dificultades burocráticas, corrupción y falta de experiencia de las empresas chinas, así como la sanción de legislaciones que restringen la venta de tierras y la emergencia de fuertes rechazos sociales (Ellis, 2015; Gooch y Gale, 2018). Si bien ello subraya el riesgo de sobrevalorar las inversiones de la potencia, no explica el significado del acaparamiento de tierras de China dentro las dinámicas de acumulación capitalista, ni, a la inversa, como los límites que emergen producto de la crisis ambiental afectan la ejecución de esos proyectos y, con ello, las posibilidades de acumulación.

Las cuestiones señaladas son prioritarias para el enfoque ecología-mundo de Jason Moore (2015a), que se aparta de la escisión entre naturaleza y humanidad para comprender cómo el capitalismo trabaja a través de la naturaleza, y cómo la naturaleza trabaja a través del capitalismo. Desde esta perspectiva, el trabajo indaga las implicancias del acaparamiento de tierras de China

Esas operaciones consisten en la adquisición de las comercializadoras de granos y aceites Noble y Nidera por parte de COFCO y la adquisición de Syngenta, líder en semillas y fertilizantes, por parte de Chemchina. 
con finalidad alimentaria alrededor del mundo sobre el proceso de acumulación capitalista. Para ello, se estudian sus inversiones en tierras para la producción de soja, arroz y caña de azúcar. Esos cultivos concentraron los intentos de China de controlar tierras en el exterior, pero, mientras que una fracción de esas operaciones fue ejecutada, otras no avanzaron más allá de la negociación o fueron abandonadas. El estudio se extiende desde el año 2000, cuando comienza el ascenso del precio internacional de los alimentos (Moore, 2012), hasta el año 2019.

El trabajo argumenta que el acaparamiento de tierras de China expresa e intensifica las contradicciones en el proceso de acumulación. Por un lado, supone una expansión de la participación del agronegocio chino en el sistema alimentario financiarizado para apropiarse de las tierras indispensables para responder a las necesidades alimentarias de sus trabajadores y su clase media. No obstante, por el otro, los altos costos que la crisis ambiental impone a esos proyectos no solo limitan su ejecución, sino además las posibilidades de una nueva expansión del capitalismo.

En términos metodológicos, es necesario destacar que no existen datos disponibles para exponer con precisión las inversiones en tierras de China (Chen et al., 2017). A menudo la información es presentada de modo fragmentado, con inconsistencias y ausente de verificación (Hofman y Ho, 20I2). Dada la imposibilidad de realizar un relevamiento exhaustivo, este trabajo analiza la evolución de las inversiones en tierras de China a partir de información extraída de Land Matrix (s.f.). Esta es una base de datos abierta que recolecta información de los acuerdos intentados, concluidos y fallidos para adquirir tierras mediante compra, arrendamiento o cesión para la producción agrícola, forestal, extractiva, el comercio de carbón, la producción de energías renovables, la conservación y el turismo.

Este trabajo se focaliza exclusivamente en las iniciativas vinculadas a la producción de soja, arroz y caña de azúcar para la alimentación, por lo que se examinan sesenta y un acuerdos de los 37I protagonizados por China. Por otra parte, dado que estos acuerdos suelen involucrar la producción de múltiples cultivos, para establecer la extensión dedicada a cada producto solo se consideran los que poseen un único cultivo. Complementariamente, se recurre a información de prensa y publicaciones académicas que permitan profundizar el análisis de ciertos casos.

El primer apartado del artículo caracteriza el acaparamiento de tierras a partir de las nociones de comida barata y hegemonía del capital financiero desde el enfoque de ecología-mundo. Introduce además el concepto de valor negativo. El segundo apartado desarrolla las razones de las inversiones en tierras de China en función de su posición como polo de acumulación global. La tercera sección se concentra en el acaparamiento de tierras de China para la producción de soja, arroz y caña de azúcar.Además de describir el desarrollo del proceso, se analiza la relevancia doméstica de esos cultivos y las causas de su producción en el exterior. Finalmente, se reflexiona sobre los límites ambientales a la acumulación de capital mediante el acaparamiento de tierras.

\section{Ecología-mundo y acaparamiento de tierras}

La escisión entre naturaleza y sociedad desde la modernidad ha sido fundamental para el desarrollo del capitalismo. Esa visión binaria en que la naturaleza es algo externo, susceptible de ser cuantificado, transformado o racionalizado para el crecimiento económico (Moore, 20I5a), 
oscurece el entendimiento de los orígenes de la actual crisis ambiental. Ese pensamiento no solo está presente en los enfoques dominantes de las Relaciones Internacionales, que ignoran la naturaleza o la tratan como exógena a los procesos sociales (Corry, 2020), sino también en la Economía Política Internacional, donde predominaron las lecturas institucionales de los problemas ambientales (Saguier y Ghiotto, 2018). El dualismo, además, es reforzado visualmente por la proliferación de imágenes que reflejan la destrucción ambiental desde marcos extractivos que restringen las alternativas (Saguier, 2019).

Para superar la disociación naturaleza/sociedad, este trabajo adopta la interpretación de Moore (20I5a) del capitalismo no como un sistema económico o social, sino como una ecologíamundo, en la que la acumulación de capital, la búsqueda de poder y la coproducción de la naturaleza forman una unidad dialéctica. Ello significa que el capitalismo es coproducido por un conjunto de relaciones en la red de la vida, lo que coloca en el centro del análisis la relación del capitalismo en la naturaleza/la naturaleza en el capitalismo. Esto posibilita observar las estrategias y relaciones de larga duración que permitieron sobrevivir al capitalismo mediante la transformación de la naturaleza en valor. Para ello, la naturaleza ha sido obligada a trabajar cada vez más intensamente de forma gratuita o a bajo costo.

Este enfoque contribuye al entendimiento del acaparamiento de tierras, que refiere al proceso de toma de control de grandes extensiones de tierras cultivables como respuesta a la demanda de recursos naturales de los nuevos polos de acumulación global y las dinámicas de acumulación del capital en un escenario de crisis (Borras et al., 20I2). Específicamente, este trabajo interpreta que el acaparamiento de tierras se enraíza en dos rasgos actuales de la ecología-mundo capitalista: el fin de la comida barata y la hegemonía del capital financiero.

En relación a la primera, Moore (2010) reconoce que las grandes olas de acumulación de capital fueron posibilitadas por la expansión de un excedente ecológico, que tuvo como núcleo a los denominados Four Cheaps. Estos son la fuerza de trabajo, los alimentos, la energía y las materias primas, considerados baratos debido a que su baja composición de valor, es decir, el tiempo de trabajo necesario para su producción permite reducir los costos de producción (Moore, 20I2). Vale resaltar que los alimentos ocupan un lugar destacado a causa de su fuerte conexión con el precio de la fuerza de trabajo. Más aún, la comida barata fue un pivote de los años dorados del capitalismo, que mediante sucesivas revoluciones agrícolas logró proporcionar alimentos baratos a un proletariado en aumento, lo que posibilitó disminuir el salario mínimo (Moore, 20I0).

Es importante subrayar que el capitalismo obtiene el excedente ecológico mediante una dialéctica de productividad y saqueo, que combina la expansión de la frontera de apropiación de las naturalezas humana y extrahumana con innovaciones científico-tecnológicas que revolucionen la productividad del trabajo. La productividad y el saqueo también motorizaron las revoluciones agrícolas, lo que demuestra que la producción de plusvalía requiere de un acto mayor de apropiación de trabajo/energía de la naturaleza no pagos. De ahí la centralidad que poseen las fronteras en la acumulación.

Justamente, Moore (2013) considera a la infinita acumulación de capital y la apropiación de la Tierra como dos caras de la misma moneda. La razón es que el capitalismo depende del 
movimiento hacia nuevas fronteras no capitalizadas que tornen disponibles de forma barata los bienes de la naturaleza humana y extrahumana. Asimismo, la producción y ocupación de nuevas fronteras permitió al capital evadir los límites provocados por el agotamiento de los agroecosistemas.

No obstante, actualmente las fronteras se han agotado (Moore, 20I5b). Los consecuentes obstáculos a la expansión espacial del capitalismo alentaron una estrategia extractiva por parte del capital financiero, que no solo avanzó sobre la vida cotidiana, sino que diseñó nuevas y más despiadadas formas para apropiarse de la naturaleza (Moore, 20I2). Ello sentó las bases para el rasgo distintivo de la acumulación global en la era neoliberal: la hegemonía del capital financiero (Moore, 20I2). Reflejo de ello es el ingreso masivo de actores financieros en el sistema alimentario global. Estos ganaron un enorme poder dentro de la cadena agroalimentaria a través del uso de productos derivados, como los mercados de futuros, que les confieren una influencia decisiva sobre las condiciones de mercado y los precios de los commodities. Con todo, también las mayores comercializadoras de granos, Archer Daniels Midland (ADM), Bunge, Cargill y Louis Dreyfus, conocidas como el grupo $A B C D$, convirtieron a las actividades financieras en un pilar de sus negocios (Clapp, 20l4).

La paradoja de las finanzas es que lograron la hegemonía no solo cuando las fronteras llegaron a su límite, sino además en el momento en que la subordinación del capital productivo obstaculizó las revoluciones científico-tecnológicas. Así, el neoliberalismo rompió la dialéctica de productividad y saqueo a favor del despojo de activos, la expoliación y las inversiones especulativas que aumentaron la volatilidad de los mercados de bienes (Moore, 20I2). Con ello se agotó la comida barata, la condición más importante para superar las crisis sistémicas.

En este marco, el progresivo encarecimiento de los alimentos desde el año 2003 y el declive de la productividad del Norte tornaron a la tierra en una nueva frontera de inversión (McMichael, 2012) para operadores financieros. Estos fueron atraídos por las expectativas de ganancias por la apreciación de la tierra y la percepción de ésta como un activo seguro y tangible frente a la inestabilidad financiera de 2008 (Cotula, 20I2). Aun así, la tierra no fue tratada solo como activo financiero, sino asimismo como un medio de producción por inversores que tras adquirir la tierra se involucraron en la producción agrícola a fin de aprovechar la rentabilidad de la actividad (Fairbain, 2012).

Lo anterior explica la atracción del capital financiero por los cultivos y materias primas flexibles, caracterizados por poseer múltiples usos —alimentación, forraje, combustibles, industriales - y ser intercambiables con flexibilidad (Borras et al., 2016). Representativa de ellos es la soja, utilizable como forraje, alimento o biodiesel; la caña de azúcar, empleada como alimento y etanol; y la palma aceitera, que tiene un fin alimentario, como biocombustible y un uso comercial e industrial. Para las finanzas, la relevancia de estos reside en su multifuncionalidad, lo que permite la diversificación y asegura la liquidez de la inversión. Además, la alta demanda de cada cultivo en el conjunto de los mercados estimula el ascenso de los precios. Los cultivos flexibles, entonces, confirman que las decisiones de producción reflejan un cálculo financiero (McMichael, 20I2).

Ahora bien, conviene subrayar que el acaparamiento de tierras no solo responde a los 
intereses del capital financiero, sino fundamentalmente al fin de la comida barata (McMichael, 2012). De ahí que haya sido impulsado asimismo por estados altamente dependientes de las importaciones de productos primarios. La participación de estos se origina en su necesidad de garantizar la seguridad alimentaria doméstica, mediante el control de tierras en otros territorios para la producción de materias primas a fin de eludir la volatilidad de los mercados.

Por consiguiente, la variedad de intereses en torno a la tierra desató una competencia para abrir las fronteras remanentes en el mundo mediante el acaparamiento de tierras a fin de apropiarse los alimentos baratos que permitan reimpulsar la acumulación. Con todo, para analizar los resultados de este proceso se incorpora el concepto de valor negativo. Moore (20l5b) lo caracteriza como la emergencia de límites al capital que restringen la restauración de los Four Cheaps. Esos límites expresan la creciente hostilidad de la naturaleza a la acumulación de capital en ausencia de nuevas fronteras. Ello origina problemas que combinan el agotamiento de recursos, altos costos de producción y la desestabilización de la biosfera. Consecuentemente, el capital debe recurrir a estrategias progresivamente costosas, tóxicas y peligrosas para fijar la naturaleza.

En definitiva, el valor negativo demuestra las contradicciones del capital en la naturaleza que son propulsadas y se expresan a través de la ley de valor. Esos límites se introducen directamente en los costos de producción y no pueden ser externalizados. Por ello, el valor negativo no es sinónimo de impactos ambientales ni de externalidades, sino que representa límites autoformados dentro de la totalidad de la ecología-mundo capitalista y sus relaciones de valor y expresan la erosión de las relaciones que directamente transforman el trabajo/energía en capital (Moore, 20I5b).

\section{El interés de China por la tierra}

El extraordinario crecimiento económico que consolidó a China como la segunda economía mundial propició el debate en torno al significado de esa trayectoria para el capitalismo. Apartándose del discurso respecto a la "amenaza china" (Broomfield, 2003), este trabajo interpreta que la consolidación de China como uno de los polos de crecimiento económico mundial se enraíza en una fase particular de la acumulación global. En esta, ciertos territorios y sectores económicos chinos son incorporados a las redes globales de producción, estructuradas por el capital transnacional (Phillips, 2009).

En efecto, la marcha de China hacia el capitalismo se caracterizó por una dependencia exportadora y un flujo de capitales y tecnologías desde el extranjero superior al de cualquier país de desarrollo tardío. Como resultado, la potencia se tornó el mayor receptor de capital extranjero del mundo detrás de Estados Unidos (Panitch y Gindin, 2013). Ello demuestra que su crecimiento económico es inescindible de las estrategias del capital transnacional que, favorecido por las reformas implementadas desde finales de la década de los setenta y aceleradas tras el ingreso a la Organización Mundial de Comercio en 200I, aterrizó en áreas sectoriales de ese país (Phillips, 2009).

Lo anterior facilitó asimismo el surgimiento de una clase capitalista en China, guiada por la aspiración de ampliar su involucramiento en el capitalismo global y abrir nuevas vías para la 
acumulación (Harris, 2018). Con esa meta, el gobierno consideró esencial que la potencia dejara de ser exclusivamente una plataforma para las inversiones extranjeras. Ello explica que, en el año 2000, adoptara la estrategia de Go Out con el objetivo de alentar activamente las inversiones de las empresas chinas alrededor del mundo (Dussel Peters, 20I3).

Es de notar que el Go Out es un pilar del enfoque de desarrollo agrícola de China que prioriza el reemplazo de la agricultura a pequeña escala por la construcción de un robusto sector de agronegocios doméstico que contribuya al desarrollo nacional, así como al acceso a recursos y mercados en el exterior (Schneider, 2017). Acorde a ello, China limitó la participación del capital transnacional en la transformación agraria a fin de promover el liderazgo de las empresas nacionales de propiedad estatal, privada o mixta. Como resultado, más de 1300 empresas chinas poseen inversiones agrícolas, forestales y pesqueras en alrededor de cien países (Gooch y Gale, 2018).

Cabe enfatizar que las tierras agrícolas adquirieron un atractivo especial dentro del Go Out debido a la conjunción de dos factores. Primero, la dificultad de cumplir con la estrategia de autosuficiencia alimentaria definida en el Libro Blanco sobre la cuestión de los cereales, publicado en 1996. Ese documento estipula el abastecimiento del $95 \%$ de las necesidades de alimentos por fuentes nacionales, mientras que para cereales como maíz, arroz y trigo, ese nivel debe alcanzar el 100\% (Zhang y Cheng, 2016). Esto se debe, por un lado, al alza en la demanda de alimentos resultante del incremento de ingresos de la población y la transición nutricional de la clase media del país hacia el consumo de proteínas y alimentos procesados. Por el otro, China solo dispone del $8,5 \%$ de la tierra cultivable y el $6,5 \%$ de las reservas mundiales de agua para alimentar al $21 \%$ de la población mundial (Hofman y Ho, 20I2). La escasez de tierras es acentuada por la acelerada urbanización, que reorientó los suelos hacia usos no agrícolas (Zha, Zhang y He, 2018). Se añade que la contaminación derivada del desarrollo industrial provocó la pérdida de 8,3 millones de hectáreas de tierras agrícolas desde 1990 (Thomas, 2013).

La respuesta de China a la brecha entre oferta y demanda de alimentos fue la importación de cultivos que exijan amplias extensiones de tierras, como soja y maíz, mientras sus agricultores se concentraron en los productos intensivos en trabajo (Zha, Zhan y He, 20l8). Con todo, el interés por la tierra agrícola fue motorizado, en segundo lugar, por su desconfianza hacia el comercio como medio para satisfacer las necesidades alimentarias. Esa aprensión se origina en la volatilidad del precio de los commodities y, especialmente, el riesgo de que su transmisión a nivel interno genere inestabilidad política y social. Lo anterior es profundizado por el dominio del grupo ABCD sobre el comercio mundial de granos. Estas empresas comercializan además el $80 \%$ de la soja importada por China (Brautigam, 20I5), lo que la coloca en una posición desventajosa en la cadena agrícola global.

La conjunción de esos factores empujó a China a proyectar su escasez doméstica de tierras hacia las fronteras externas restantes, mediante la inversión en grandes extensiones de tierras para el outsourcing de la producción agrícola (Hofman y Ho, 20I2). Mientras que Gran Bretaña, en el siglo XIX, dislocó su agricultura hacia el Nuevo Mundo para proveer alimentos baratos a la mano de obra en Europa, la particularidad de China es que externaliza su agricultura para satisfacer las exigencias alimentarias de su creciente clase media y para asegurar los alimentos de 
sus trabajadores (McMichael, 2019). Ello es central para mantener los salarios industriales bajos y la competitividad internacional de las manufacturas chinas (Puyana y Costantino, 20I5). No obstante, el acceso a alimentos baratos es inescindible del objetivo de promover la expansión global del agronegocio chino para incrementar su poder en el sistema alimentario financiarizado, a fin de competir con las grandes comercializadoras de granos (McMichael, 2019).

\section{El despliegue del acaparamiento de tierras de China}

Land Matrix (2020) posiciona a China como el mayor inversor en tierras del mundo. Esa fuente reporta 37I acuerdos por tierras protagonizados por empresas chinas, que involucran conjuntamente I8.737.456 hectáreas ${ }^{2}$. De ese total, I I4 acuerdos se vinculan estrictamente a la producción de alimentos, orientados a diversos cultivos como maíz, trigo, frutas, vegetales, castañas, yuca y aceite de palma. Sin embargo, al considerar las hectáreas involucradas en esos tratos, salta a la luz la prioridad de la soja en la búsqueda de tierras, con iniciativas que abarcaron una extensión de 750.100 hectáreas. Detrás de ella se encuentra el arroz, con 189.029 hectáreas y la caña de azúcar, con 125.272 hectáreas $^{3}$. Los próximos apartados exploran en detalle el acaparamiento de tierras de China para el acceso a esos cultivos.

\section{I. El acaparamiento de tierras para la producción de soja}

La soja posee una importancia primordial para China. El país es el mayor consumidor mundial de la oleaginosa, que es clave para producir carne de soja utilizada como forraje en la producción porcina y avícola industrial. Esta actividad fue estimulada desde fines de la década de los setenta, cuando las autoridades se esforzaron por modernizar las dietas, lo que implicaba incrementar el consumo de carne de las clases medias y altas. El desarrollo del sector de forrajes se tornó indispensable para esa meta.Vale destacar que, actualmente, China lidera la producción mundial de forrajes, de carne de cerdo y aves de corral, y de aceite de soja para consumo humano (Oliveira y Schneider, 2016).

En el siglo $\mathrm{XXI}$, China modificó su rol de exportador neto de soja para convertirse en el mayor importador global ${ }^{4}$. La contracara de ello fue el declive de la producción doméstica, que afectó especialmente a Heillongjiang, la principal provincia productora, donde el área sembrada con este producto disminuyó un 42\% (Hairong, Yiyuan y Bun, 2016). A esto también contribuyó la diferencia entre el precio de mercado de la soja y los crecientes costos de mano de obra para la producción, que estimuló a los agricultores a volcarse hacia cultivos de alto valor, como carne, vegetales y frutas (J. Zhang, 2019). Cabe añadir que estos encuentran dificultades para competir con el bajo precio de la soja genéticamente modificada importada, cuyo cultivo doméstico fue prohibido. La consecuencia de esto es que el $30 \%$ de los pequeños productores de soja se vio obligado a buscar trabajo en las ciudades (Oliveira y Schneider, 20 I6).

2 Detrás de China se encuentra Estados Unidos, con 340 acuerdos por 13.214.439 hectáreas, seguido por Canadá, con 233 acuerdos por II.004.623 hectáreas y Reino Unido, con 316 acuerdos por 10.851.008 hectáreas.

3 Para calcular esa extensión solo se consideran los proyectos que involucran exclusivamente la producción de cada uno de esos cultivos.

4 Ello fue facilitado por la liberalización de las importaciones de soja antes del ingreso a la Organización Mundial de Comercio y la decisión del gobierno de redefinir la soja como un cultivo industrial, lo que la excluyó de los controles de precios (McMichael, 2009). 
En este contexto, China concentró sus importaciones de soja en Sudamérica, lideradas por Brasil — que junto a Argentina abastece más de la mitad de su demanda-, y en Estados Unidos, el segundo proveedor de la oleaginosa. No obstante, el dominio de los traders sobre las importaciones chinas de soja y el ascenso de los precios de los commodities ponían en riesgo su acceso al cultivo. Esto impulsó a las empresas chinas a adquirir tierras alrededor del globo para dedicarlas a la producción de soja. Adicionalmente, ese objetivo representaba una oportunidad para las procesadoras chinas de soja, interesadas en compensar su exceso de capacidad (Oliveira y Schneider, 2016).

Como resultado, América Latina se convirtió en una frontera clave para que el gigante asiático disloque la producción de soja. Una razón es la aptitud de esa región para los cultivos intensivos en agua, en contraste con la disminución de las reservas y la desertificación en el norte y el noreste de China (McMichael, 2019). Los datos exponen la existencia de ocho proyectos de empresas chinas, seis de los cuales les permitirían controlar 749.600 hectáreas para dedicarlas con exclusividad a ese cultivo.

Tabla I: Inversiones chinas en tierras para la producción de soja

\begin{tabular}{|c|c|c|c|c|}
\hline Hectáreas & Estado & Inversor & País & Cultivo \\
\hline 320.000 & Suspendido & Beidahuang Group & Argentina & soja \\
\hline 200.000 & Suspendido & Chongqing Grain & Brasil & Soja \\
\hline 200.000 & Sin datos & Shangai Pengxing Group & Brasil & soja \\
\hline 126.000 & En operación & Dongjin Group & Rusia & Soja-granos \\
\hline 60.000 & Suspendido & Beidahuang Group & Venezuela & Maiz-arroz- soja \\
\hline 5772 & En operación & Eximbank & Rumania & $\begin{array}{c}\text { Alfalfa-cebada-colza- } \\
\text { soja-girasol-trigo }\end{array}$ \\
\hline 35.000 & En operación & Dongjing Group & Rusia & $\begin{array}{c}\text { Soja- forrajes- } \\
\text { ganadería }\end{array}$ \\
\hline 21.900 & En operación & Fu Yuan Jin Liang Modern & Rusia & $\begin{array}{c}\text { Soja-forrajes- } \\
\text { ganadería }\end{array}$ \\
\hline 16.000 & En operación & Zhejiang Fudi Agriculture Co. & Brasil & Soja \\
\hline 13.000 & Sin datos & Chonqing Grain & Argentina & Soja \\
\hline 12.500 & En operación & Shanghai Pengxin Group & Bolivia & Maíz-sorgo-soja \\
\hline 10.000 & En operación & Anhui State Farms Group & Zimbawbwe & Maíz-soja-trigo \\
\hline 3500 & En operación & Beidahuang Group & Rusia & Maíz-soja-trigo \\
\hline 2549 & En operación & Chenguang Biotech & Zambia & Maíz-soja-trigo \\
\hline 612 & Abandonado & HuaYong Overseas Group & Zambia & Maíz-soja- vegetales \\
\hline 600 & En operación & Zhejiang Fudi Agriculture Co. & Brasil & Soja \\
\hline 500 & Abandonado & JiangxiYu Sheng Food & Ghana & soja \\
\hline 62 & En operación & Chongqind Seed Corp & Tanzania & Maíz-oleaginosas-arroz \\
\hline
\end{tabular}

Fuente: Elaboración propia en base a Land Matrix (2020) 
Argentina y Brasil fueron los principales destinos de esas iniciativas. En la primera, el ejemplo más importante es el convenio firmado por la empresa estatal Beidahuang Group con la provincia de Río Negro para la instalación de riego y la construcción de una terminal portuaria (Murmis y Murmis, 2012). Sin embargo, ese acuerdo transfería el control de más de 320.000 hectáreas de valles irrigables a la empresa mediante una combinación de arrendamiento por veinte años y cesión de tierras para la producción de soja para exportar a China. Ese cultivo motivó, además, una compra de tierras de menor escala por la estatal Chonqing Grain en la provincia de Santiago del Estero.

Por su parte, una de las inversiones chinas movilizadas por la soja con mayor resonancia en Brasil fue el intento de Chongqing Grain de comprar 200.000 hectáreas en el estado de Bahía (Oliveira, 2017). Similar interés guío la adquisición de 600 hectáreas en el estado de Río Grande del Sur y la compra de 16.000 hectáreas en el estado de Tocantins por las empresas Zhejiang Fudi Agriculture Company y Beidahuang Group. Land Matrix también menciona negociaciones de la empresa Shangai Pengxin Group para comprar 200.000 hectáreas en ese país.

A los anteriores se añaden dos proyectos que, aunque combinaron varios cultivos, reafirman la relevancia de la región para el aprovisionamiento chino de soja. Por un lado, la compra de I 2.500 hectáreas en la región de Santa Cruz de la Sierra en Bolivia por Shanghai Pengxin Group para sembrar maíz, sorgo y soja. Por el otro, la firma de un convenio entre Beidahuang Group y el gobierno venezolano, en $20 \mathrm{II}$, para capacitación, infraestructura, tecnología y financiamiento en la producción de alimentos (PSUV, 20II), que incluía la adquisición de 60.000 hectáreas para cultivar maíz, soja y arroz.

Asia se convirtió en la segunda frontera para la soja, con cuatro operaciones ubicadas en Rusia.Aunque no es posible precisar la cantidad de hectáreas involucradas, hay que destacar que el este del país se convirtió en una frontera privilegiada para las inversiones agrícolas chinas debido a los bajos costos de los arrendamientos y salarios y el precio del combustible. Esto contrasta con el reducido nivel de importaciones por cuestiones impositivas, climáticas y problemas de infraestructura (Gooch y Gale, 2018). Además, los agricultores de Heilongjiang y Jinlin, provincias limítrofes con Rusia que forman parte del cinturón de tierra negra, encuentran condiciones climáticas e hidrológicas similares para trasladar sus actividades a ese país (Zhou, 2017).

La empresa privada Dongjing Group se destaca por sus proyectos para producir soja en Rusia. Entre ellos se encuentra el arrendamiento de 53.000 hectáreas, de las que destinaría 13.000 al cultivo de soja (China Daily, 2017).A esa operación, que convirtió a China en el mayor inversor en el sector agrícola ruso, se añade un joint venture, que actualmente posee 35.000 hectáreas en operación (Zhou, 20I7). En cambio, además de acceder a soja, Fu Yuan Jin Liang Modern Agriculture LLC se interesó por la ganadería, los forrajes y los lácteos (East Russia, 2019). Beidahuang Group fue otra empresa atraída por las tierras rusas, aunque solo opera 3500 hectáreas.

En contraste, en África la soja estuvo presente en cinco acuerdos por tierras, cuatro de los cuales incluyeron otros granos. Solo 500 hectáreas en Ghana son dedicadas al cultivo exclusivo de soja producto del arrendamiento de la empresa Jiangxi Yu Sheng Food. Mientras tanto, en Europa del Este, la soja provocó una operación financiada por el Banco de Exportaciones e Importaciones 
de China (Eximbank) por el arrendamiento de 5770 hectáreas.

\subsection{El acaparamiento de tierras para la producción de arroz}

China es el mayor productor y consumidor mundial de arroz. Este es el alimento básico de más del $65 \%$ de su población, así como un cultivo de subsistencia para la mayoría de los agricultores de bajos recursos en las zonas rurales (Peng et al., 2009). Por esa razón, la autosuficiencia en la producción de arroz ha sido un pilar de la estrategia de seguridad alimentaria del país. Aun así, la transición nutricional provocó una disminución en el consumo de ese grano, en especial en los hogares de mayores ingresos, que se prevé que se profundizará en el futuro.

No obstante, este proceso es paralelo a un rápido incremento de las compras externas de arroz, que convirtieron a China en el mayor importador mundial en 2012. En primer lugar, ese resultado responde a la necesidad de China de satisfacer la demanda por variedad de alimentos de la población. Reflejo de ello es el alza del consumo de variedades de arroz provenientes de Vietnam, Camboya, Pakistán y Tailandia, que compiten con los cultivadores chinos en precio, calidad y seguridad (Xu, Su y Lone., 20l8). La última es sumamente relevante, ya que las importaciones fueron estimuladas, además, por la preocupación social que generaron los escándalos por la producción de arroz tóxico (H. Zhang, 20l9).

El incremento de las importaciones es provocado, en tercer lugar, por la suba de los precios de compra fijados por el gobierno chino a fin de impulsar la producción doméstica de arroz. Entre 2007 y 20I4, estos se duplicaron (H. Zhang, 2019), lo que perjudicó a los consumidores de menores ingresos. Esto incentivó las importaciones de arroz de menor calidad de Laos, India y Camboya (Xu, Su y Lone, 2018). En adición, es probable que el alza de los costos de producción, la competencia de cultivos y la escasez de agua aumenten el atractivo del arroz importado (Bandumula, 2017).

El control de tierras en el exterior para la producción de arroz barato, por lo tanto, constituye una forma de reemplazar y reducir las importaciones de un cultivo tan valioso para la seguridad alimentaria del país. Pero además de proteger al arroz de las vicisitudes de los mercados, este proceso es alentado por el interés de China en proyectar globalmente sus empresas multinacionales de semillas. Estas disponen de variedades de arroz híbrido resultantes de décadas de investigación, además de las condiciones climáticas y la mano de obra necesarias para su desarrollo. Ello brindaría a China un mayor control de la producción de arroz más allá de las fronteras para garantizar su abastecimiento doméstico, como también el de sus trabajadores en megaproyectos alrededor del mundo (GRAIN, 2008).

Las empresas chinas dirigieron la mayoría de sus inversiones en tierras para el abastecimiento de arroz hacia África, que registró trece acuerdos. Ahora bien, este fue el cultivo exclusivo de nueve iniciativas, que suman un total de 84.09I hectáreas. Entre los proyectos de mayor extensión se encuentra la construcción y operación de una granja de arroz de 35.000 hectáreas y una plantación de caucho de 100.000 hectáreas en Sierra Leona mediante un joint venture entre China Hainan Company for International Economic Cooperation y Jiangsu Provincial Construction Group (Brautigam, 2015). Igualmente relevante es la iniciativa de la empresa privada Wanbao 
Grains y Oils para desarrollar sistemas de irrigación en la provincia de Gaza, en Mozambique, que exigió una concesión de 20.000 hectáreas de tierra cultivable por 50 años (Mora, 2016).

Vale destacar que el arroz también motivó operaciones de menor escala. Ejemplo de ello son los arrendamientos de 150 hectáreas en Madagascar y 766 hectáreas en Uganda. La base de datos también reporta dos operaciones de Chonqing Seed Corp para el cultivo en 300 hectáreas en Nigeria y Tanzania respectivamente.

Tabla 2: Inversiones chinas en tierras para la producción de arroz

\begin{tabular}{|c|c|c|c|c|}
\hline Hectáreas & Estado & Inversor & País & Cultivo \\
\hline 1.000 .000 & Suspendido & Jilin Fuhuan Corp. & Filipinas & Maíz-arroz-sorgo \\
\hline 200.000 & Sin datos & Beidahuang Group & Filipinas & Maíz- arroz \\
\hline 135.000 & Sin datos & $\begin{array}{c}\text { China Hainan Company } \\
\text { for International Economic } \\
\text { Cooperation }\end{array}$ & Sierra leona & Arroz \\
\hline 20.000 & En operación & Wanbao & Mozambique & Arroz \\
\hline 20.000 & En operación & $\begin{array}{c}\text { Yunnan Jin Chen Investment } \\
\text { Company }\end{array}$ & Myanmar & $\begin{array}{l}\text { Café-maiz-arroz- } \\
\text { sesamo }\end{array}$ \\
\hline 17.500 & Suspendido & Yuan lonping & Zambia & Arroz \\
\hline 10.003 & Sin datos & Hunan Yuan International & Madagascar & Arroz \\
\hline 8900 & En operación & $\begin{array}{l}\text { State Development and } \\
\text { Investment Corp }\end{array}$ & Madagascar & $\begin{array}{c}\text { Caña de azúcar- } \\
\text { arroz }\end{array}$ \\
\hline 8000 & Suspendido & $\begin{array}{c}\text { China National Corporation } \\
\text { for Overseas Economic } \\
\text { Cooperation }\end{array}$ & Camboya & $\begin{array}{l}\text { Maiz-arroz } \\
\text {-vegetales }\end{array}$ \\
\hline 5000 & Sin datos & Suntime & Cuba & Arroz \\
\hline 5000 & Suspendido & Chongqing government & Laos & $\begin{array}{c}\text { Arroz híbrido- } \\
\text { acuicultura }\end{array}$ \\
\hline 4000 & Suspendido & Liu Jianjun & Uganda & Maiz- arroz \\
\hline 776 & En operación & $\begin{array}{l}\text { Zhong Yi International } \\
\text { Agriculture Comp. }\end{array}$ & Uganda & Arroz \\
\hline 303 & Sin datos & Sichuan & Uganda & Frutas-arroz \\
\hline 300 & En operación & Chongqing Seed Corp & Nigeria & Arroz \\
\hline 300 & Suspendido & Chonqing seed & Tanzania & Arroz \\
\hline 150 & Suspendido & $\begin{array}{c}\text { Weichu Madagascar Agricultural } \\
\text { Development }\end{array}$ & Madagascar & Arroz \\
\hline 62 & En operación & Chongqing Seed Corp & Tanzania & $\begin{array}{c}\text { Maíz-oleaginosas- } \\
\text { arroz }\end{array}$ \\
\hline- & Sin datos & Tan Yifei & Malasia & Arroz \\
\hline- & Sin datos & Sang liang & Nigeria & Arroz \\
\hline
\end{tabular}

Fuente: Elaboración propia en base a Land Matrix (2020) 
Las tierras del sudeste asiático fueron otra frontera para la producción de arroz, con seis proyectos. Todos combinaron diferentes cultivos, con excepción de la empresa Tian Yifei, que expresó su interés en establecer un joint venture con un gobierno estatal en Malasia para desarrollar una plantación comercial de arroz. Entre los restantes acuerdos se encuentra el proyecto de parque agrícola del gobierno municipal de Chongqing, en Laos, con el objetivo de producir arroz, flores y acuicultura en 5000 hectáreas (Gooch y Gale, 2018). De modo similar, Yunnan Jin Chen Investment Company invirtió en 20.000 hectáreas en Myanmar para producir caucho y otros cultivos (Woods y Kramer, 2012). Por su parte, China National Corporation for Overseas Economic Cooperation intentó arrendar 20.000 hectáreas en Camboya.

Es importante destacar que la producción de arroz híbrido, maíz y sorgo propició el intento de acaparamiento de tierras de mayor extensión en Asia. Este ocurrió en 2007, cuando el Gobierno de la provincia china de Jilin, el Banco de Desarrollo de China y jilin Fuhua Agricultural Science and Technology Development Co. firmaron con el Gobierno de Filipinas un acuerdo de cooperación en granos genéticamente modificados. Aunque se iniciaría en 50.000 hectáreas, fue previsto que las partes chinas arrendaran I millón de hectáreas sin explotar, lo que equivalía al $10 \%$ de las tierras cultivables de Filipinas. El emprendimiento fue suspendido luego de que se cuestionara legalmente la transferencia de esa extensión de tierras (Thomas, 20I3). En ese país tuvo lugar otro proyecto ambicioso que no prosperó: el arrendamiento de 200.000 hectáreas por parte de Beidahuang Group.

Finalmente, el acaparamiento de tierras para la producción de arroz tiene un solo ejemplo en América Latina. Este ocurrió en 1996, cuando Suntime Group arrendó 5000 hectáreas en Cuba para brindar asistencia en la producción del grano (Myers y Jie, 20I6).

\subsection{El acaparamiento de tierras para la producción de caña de azúcar}

China es el tercer productor mundial de azúcar detrás de Brasil e India, con más del $90 \%$ de ese total originado en la caña de azúcar (Li y Li-Tao, 20I5). Esta es esencial para abastecer el creciente consumo de azúcar de la población, estimulado por el cambio en las preferencias y la demanda de alimentos procesados, bebidas carbonatadas y jugos. Pese a que los altos precios y el reemplazo por edulcorantes explican que China posea un consumo per cápita anual de azúcar de $10,5 \mathrm{~kg}$, sustancialmente menor al promedio mundial de $17 \mathrm{~kg}$ (USDA, 2019), se espera que este se incremente.

Con todo, es importante mencionar que China es el mayor importador mundial de caña de azúcar, con Brasil como principal abastecedor. La dinamización de las compras externas responde, por un lado, a que la producción doméstica de azúcar no es suficiente para satisfacer la demanda (Li y Li-Tao, 20I5). Por el otro, a la diferencia de precios entre los mercados doméstico e internacional. La razón es que el sector azucarero chino se caracteriza por la producción en pequeña escala y los altos costos de mano de obra, originados en la migración de los trabajadores rurales hacia las ciudades en búsqueda de mayores ingresos. A ello se suma el ascenso del precio de los fertilizantes y pesticidas, que provocó que los agricultores cambien de cultivo según la rentabilidad. En consecuencia, los costos totales de la producción azucarera china casi duplican a los de otros países (USDA, 2019). De ahí que el Gobierno haya calificado el auge de las importaciones 
como una amenaza a la industria azucarera nacional (Bloomberg, 2020).

Por consiguiente, el acaparamiento de tierras se tornó fundamental para prescindir de las importaciones y acceder a caña de azúcar producida con mano de obra de menor costo y en tierras más aptas. Es de notar que la productividad de la caña de azúcar en China está en declive a causa de las sequías ( $\mathrm{Li}$ y $\mathrm{Li}$-Tao, 20I5), mientras que la urbanización, la competencia con la producción de frutas y vegetales y la falta de mecanización obstaculizan la expansión del cultivo en su territorio (USDA, 2019). Adicionalmente, los bajos costos de la producción en el exterior beneficiarían a la industria del procesamiento, que en Guangxi, la principal productora de caña de azúcar, se compone de 104 molinos de azúcar pertenecientes a treinta empresas, entre ellas cinco empresas estatales chinas ( $\mathrm{Li}$ y Li-Tao, 20I5).

África se convirtió en la frontera predilecta para la producción de caña de azúcar, que motivó nueve acuerdos, con 70.264 hectáreas destinadas exclusivamente a ese cultivo. Uno de los destinos fue Benín, donde la estatal China National Complete Plant Import y Export Corporation (COMPLANT) arrendó 5200 hectáreas por 25 años.Asimismo, esa empresa adquirió dos complejos azucareros por 14.064 hectáreas y arrendó otras 6000 hectáreas en Madagascar (Brautigam, 20I5).

Tabla 3: Inversiones chinas en tierras para la producción de caña de azúcar

\begin{tabular}{|c|c|c|c|c|}
\hline Hectáreas & Estado & Inversor & País & Cultivo \\
\hline 200.000 & Sin datos & Wuhan Kaidi & Myanmar & Maíz, jatropha, caña de azúcar \\
\hline 25.000 & Suspendido & Longping Group & Etiopía & Caña de azúcar \\
\hline 23.265 & En operación & CyC Mining & Camerún & Maíz-caña de azúcar-jatropha \\
\hline 20.000 & En operación & CLECT & Malí & Caña de azúcar \\
\hline 18.000 & En operación & COMPLANT & Jamaica & Caña de azúcar \\
\hline 14.064 & En operación & COMPLANT & Madagascar & Caña de azúcar \\
\hline 9700 & En operación & Ve Wong Corp & Camboya & Caña de azúcar \\
\hline 9400 & En operación & Ve Wong Corp & Camboya & Caña de azúcar \\
\hline 9059 & Sin datos & Great Field & Camboya & Caña de azúcar \\
\hline 8900 & En operación & COMPLANT & Madagascar & Caña de azúcar-arroz \\
\hline 8849 & Sin datos & Yellow field & Camboya & Caña de azúcar \\
\hline 6000 & En operación & COMPLANT & Madagascar & Caña de azúcar \\
\hline 5200 & Suspendido & COMPLANT & Benín & Caña de azúcar \\
\hline 1845 & En operación & COMPLANT & Sierra Leona & Yuca-caña de azúcar \\
\hline- & Suspendido & Viscount Energy & Nigeria & Yuca-caña de azúcar \\
\hline
\end{tabular}

Fuente: Elaboración propia en base a Land Matrix (2020)

Por otra parte, en Malí, China Light Industrial Corporation for Foreign Economic and Technical Cooperation (CLETC) adquirió 20.000 hectáreas para la construcción de un proyecto azucarero que actualmente opera a baja capacidad porque solo fueron cedidas 3570 hectáreas. Mientras tanto, en Etiopía, la empresa Hunan Dafengyuan Agricultural Company intentó arrendar 25.000 hectáreas (Brautigam, 20I5). 
En el caso de Asia, la caña de azúcar impulsó cinco transacciones por tierras, con cuatro de ellas concentradas en el cultivo, lo que representa un total de 37.008 hectáreas. Notoriamente, todas se focalizaron en Camboya, uno de los países en que la presencia china en agricultura se expandió más aceleradamente (Thomas, 2013). Esas operaciones fueron encabezadas por la taiwanesa Ve Wong Corporation que acaparó dos plantaciones de 9400 y 9700 hectáreas. En el mismo país, las empresas Yellow Field International y Great Field firmaron acuerdos por el arrendamiento de 8849 y 9059 hectáreas respectivamente (Gooch y Gale, 20 I8).

América Latina y el Caribe tuvo una participación mínima en el acaparamiento de tierras para la producción de caña de azúcar. Esta es resultado de una operación, el arrendamiento, por parte de COMPLANT, de 18.000 hectáreas en Jamaica.

\section{El valor negativo del acaparamiento de tierras}

Una particularidad del acaparamiento de tierras de China ampliamente señalado por la literatura es el significativo número de proyectos que nunca llegaron a ser concretados (Brautigam, 20I5; Myers y Jie, 2016; Oliveira, 2017). Las inversiones analizadas en este trabajo refuerzan esa conclusión. De dieciocho iniciativas para la producción de soja, solo once entraron en operación. De modo similar, fueron ejecutados seis acuerdos para la producción de arroz de un total de veinte, mientras que solo nueve de quince iniciativas vinculadas a la caña de azúcar están en operación.

El alto porcentaje de proyectos suspendidos no puede considerarse un indicador del bajo interés de China por el control de esas tierras. Por el contrario, coloca en primer plano los límites a la acumulación de capital que son inherentes al acaparamiento de tierras. De esa forma, los altos costos que involucran estas iniciativas, a punto de obstaculizar su objetivo principal, el acceso a alimentos baratos, se tornan centrales para comprender el fracaso de esas inversiones.

Esos costos son expresión del valor negativo que emerge del acaparamiento de tierras. Una de sus formas más apremiantes se enraíza en la crisis ambiental. La localización de los proyectos en las fronteras externas remanentes obliga a las empresas chinas a internalizar el deterioro ambiental presente en esas áreas, lo que incrementa los costos. Muestra de ello es el abandono de los dos proyectos más extensos en la frontera sojera por excelencia, América del Sur. Para producir soja en 320.000 hectáreas en Argentina, Beidahuang Group debía enfrentar la extrema aridez de la Patagonia, que limita ese cultivo. Ello exigía el desarrollo de semillas que se adapten a los suelos y la expansión de los sistemas de riego. Asimismo, Chongqing Grain abandonó las negociaciones por el arrendamiento de 200.000 hectáreas en Bahía, Brasil, y firmó un contrato por el control de una hacienda más barata de 51.826 hectáreas. No obstante, la empresa tuvo dificultades con la producción agrícola, entre ellas que la tierra no estaba preparada para el cultivo y la escasez de lluvias. Además fue multada por deforestación ilegal (Oliveira, 20 I8).

Esos límites también se presentaron en uno de los mayores proyectos para la producción de arroz en África, el arrendamiento de 20.000 hectáreas por Wanbao en Mozambique. En este caso, la presencia de inundaciones provocó una demora en el financiamiento del proyecto para 
reevaluar los riegos. Recíprocamente, la pobre calidad de las tierras fue objetada por CLECT en sus operaciones en Madagascar y desalentó el arrendamiento de 25.000 hectáreas de Hunan Dafengyuan en Etiopía (Brautigam, 20I5).

Conviene subrayar que una meta del acaparamiento de tierras es establecer la agricultura industrial a gran escala, lo que exige transformar las tierras mediante la tecnificación para aumentar su productividad (McMichael, 20 I2). Esto no representa un desafío para China dada su experiencia en el desarrollo de su sector agrícola mediante la agricultura intensiva, el consumo del $30 \%$ de los fertilizantes y pesticidas del mundo, la expansión del riego y la adopción de semillas de alto rendimiento (Zhang y Cheng, 2016). Sin embargo, el intento de superar los límites que la crisis ambiental impone al acaparamiento de tierras conlleva una intensificación del uso de insumos industriales, lo que incrementa no solo los costos, sino también la toxicidad y la destructividad de esas inversiones.

Por consiguiente, no solo la degradación ambiental representa un límite al acaparamiento de tierras, sino que, a la inversa, el acaparamiento de tierras acentúa la degradación de la naturaleza, reduciendo las posibilidades de acumulación de capital. Contradictoriamente, la aplicación de fertilizantes, semillas modificadas y agroquímicos tampoco garantiza la efectividad de los proyectos, ya que las condiciones biofísicas locales también actúan como un límite al capital. Ejemplo de ello es el proyecto de parque agrícola en Laos, donde el clima local y el suelo demostraron ser poco aptos para las semillas de arroz híbridas traídas desde Chongqing, que tardó años en hallar una variedad con buen rendimiento (Gooch y Gale, 2018). Similarmente, el financiamiento de la granja de arroz en 35.000 hectáreas en Sierra Leona fue demorado a causa de la expansión de la epidemia de Ébola en ese país (Brautigam, 2015).

Los límites señalados arrojan serias dudas sobre la efectividad del acaparamiento de tierras para acceder a alimentos baratos. Más aún, esos costos son ampliados por la carencia de infraestructura en los países destino de las operaciones. De hecho, el abandono del proyecto de Wuhan Kaidi en Zambia se vinculó a que debía realizar una inversión que sería difícil de recuperar en sistemas de irrigación, carreteras, alojamiento para los trabajadores y una planta de procesamiento (Brautigam, 20I5). De modo similar, el parque agrícola en Laos fue cancelado porque demandaba inversiones en irrigación, carreteras y canales, que eran inconsistentes con las ganancias esperadas (Gooch y Gale, 2018). Más aún, Hunan Dafengyuan encontró que no podía desarrollar la tierra en Etiopía por la inexistencia de carreteras para acceder a ellas (Brautigam, 2015).

\section{Conclusión}

El acaparamiento de tierras expone el modo en que el capitalismo es coproducido por la naturaleza en un contexto de crisis, signado por el fin de la comida barata y el avance de las finanzas sobre el sistema alimentario. Por consiguiente, si bien este trabajo se focalizó en China, es necesario enfatizar que su participación en el acaparamiento de tierras es un reflejo de dinámicas inherentes al desarrollo del capitalismo tras su consolidación como un polo de acumulación global. 
En efecto, el estudio de las inversiones en tierras de China demuestra que el objetivo principal de esas iniciativas fue la apertura de fronteras en otros territorios que le permitieran superar sus limitaciones biofísicas a la producción agrícola, a fin de garantizar el acceso a alimentos baratos. Con todo, otro factor determinante de esos proyectos fue la desconfianza de China hacia los mercados agrícolas altamente concentrados y financiarizados. Esto explica que, además del arroz, las empresas chinas hayan concentrado su interés en la producción de soja y caña de azúcar, dos cultivos altamente codiciados por las finanzas, pero a fin de emplearlos con un fin alimentario.

En consecuencia, América Latina y el este de Rusia se convirtieron en las fronteras por excelencia para la producción de soja. En cambio, la producción de arroz y caña de azúcar se concentró en África y Asia. Esos cultivos son esenciales tanto para responder a la demanda alimentaria de la clase media como para disminuir el costo de los salarios de los trabajadores a fin de incrementar la productividad de las industrias. Sin embargo, el acceso a esos alimentos es indisociable de la expansión global de las empresas chinas para incrementar su poder en el sistema alimentario y, con ello, abrir nuevas vías para la acumulación. llustrativo de ello es la centralidad de la soja para mantener el liderazgo global en la producción porcina y avícola, así como las oportunidades que la difusión del arroz híbrido y el acceso a caña de azúcar barata brindarían a las empresas de semillas y la industria del procesamiento respectivamente. Esto demuestra la unidad entre acumulación, poder y naturaleza que define a la ecología-mundo.

No obstante, el acceso a alimentos baratos es obstaculizado por los límites inherentes al acaparamiento de tierras originados en la crisis ambiental. La necesidad de convertir tierras degradadas o no aptas hacia la producción agrícola exige intensificar la aplicación de tecnologías e insumos cada vez más tóxicos y destructivos, lo que incrementa extraordinariamente los costos de los proyectos. Más grave aún, en ocasiones ni siquiera el empleo de esas técnicas es suficiente para controlar las resistencias de la naturaleza al capital, en la forma de eventos climáticos como sequías e inundaciones, epidemias, o falta de aptitud de los suelos a cultivos híbridos. En definitiva, la devastación ambiental intrínseca al acaparamiento de tierras exacerba las contradicciones del capitalismo en un contexto en que la ausencia de nuevas fronteras, obstaculiza las posibilidades de acumulación.

Finalmente, un entendimiento más cabal de la efectividad del acaparamiento de tierras para acceder a naturalezas baratas requiere que investigaciones futuras extiendan el estudio del fenómeno hacia otros cultivos y actores, e incorporen iniciativas con finalidades no alimentarias. Además, los costos del acaparamiento de tierras de China orientado a alimentos tornan relevante la indagación respecto a la apertura de nuevas fronteras, como el control de la cadena de valor agrícola. Por otra parte, se requieren estudios más rigurosos de las formas en que el acaparamiento de tierras activa el valor negativo mediante el estudio de casos específicos, que además contribuyan a develar otras formas que asumen esos límites.

\section{Referencias}

Bandumula, N. (2017). Rice Production in Asia: Key to Global Food Security. Proceedings of the National Academy of Sciences, India, Section B: biological sciences, 88 (4), I 323-I 328.

Borras, S., Franco J., Isakson, R., Levidow, L. y Vervest, P. (2016). The rise of flex crops and commodities: implications for research. The Journal of Peasant Studies, 43 (I), 93-I I 5. 
Borras, S., Franco, J., Gomez, S., Kay; C. y Spoor, M. (2012). Land grabbing in Latin America and the Caribbean. The Journal of Peasant Studies, 39 (3-4), 845-872.

Brautigam, D. (20I5). Will Africa feed China?. Oxford University Press.

Broomfield, E. (2003). Perceptions of Danger:The China threat theory. Journal of Contemporary China, 12 (35), $265-284$.

Chen, Y., Li, X., Wang L. y Wang, S. (20I7). Is China different from other investors in global land acquisition? Some observations from existing deals in China's Going Global Strategy. Land Use Policy, (60), 362-372.

China Daily (16.08.2017). China becomes largest investor in Russian agricultural sector. Recuperado de: http:// subsites.chinadaily.com.cn/regional/harbin/20I7-08/I6/c |45505.htm (|4.09.2020).

Clapp, J. (20I4). Financialization, distance and global food politics. The Journal of Peasant Studies, 4I (5), 797-8I4.

Corry, O.(2020). Nature and the international:towards a materialist understanding of societal multiplicity. Globalizations, $17(3), 419-435$.

Cotula, L. (20I2). The international political economy of the global land rush: A critical appraisal of trends, scale, geography and drivers, The Journal of Peasant Studies, 39 (3-4), 649-680.

Dussel Peters, E. (2013). Características de la inversión extranjera directa china en América Latina. En: Dussel Peters, E. (Coord.). América Latina y El Caribe - China. Economía, Comercio e Inversiones (I7I-202). Unión de Universidades de América Latina y el Caribe.

East Russia (06.01.2019) How much Mu in the Far East. Recuperado de https://en.eastrussia.ru/material/pochem-muna-dalnem-vostoke/ (I4.09.2020).

Ellis, E. (20I5). Las iniciativas por parte de las firmas agrícolas chinas para establecer su presencia en América Latina y el Caribe. En: León de la Rosa, I. y Gachúz May, J.C. (Eds). Política Exterior China: relaciones regionales y cooperación (pp. 307-336). Benémerita Universidad Autónoma de Puebla.

Fairbairn, M. (20I4). 'Like gold with yield': evolving intersections between farmland and finance. The Journal of Peasant Studies, 4 I (5), 777-795.

Gooch, E. y Gale, F. (2018). China's Foreign Agriculture Investments. US Department of Agriculture, Economic Research Service, Economic Information Bulletin No. (ElB-192).

GRAIN (2008). The food crisis and the hybrid rice surge. Recuperado de https://www.grain.org/en/article/665-thefood-crisis-and-the-hybrid-rice-surge (14.09.2020)

Hairong, Y., Yiyuan, C. y Bun, K. H. (2016). China's soybean crisis: the logic of modernization and its discontents. The Journal of Peasant Studies, 43 (2), 373-395.

Harris, J. R. (2018). Who Leads Global Capitalism ? The Unlikely Rise of China. Class, race \& corporate power, 6 (I), $|7||-| 726$.

Hofman, I. y Ho, P. (20 I 2). China's “Developmental Outsourcing”:A critical examination of Chinese global “land grabs” discourse. Journal of Peasant Studies, 39 (I), I-48.

Land Matrix (s.f.). Recuperado de: https://landmatrix.org/ (14.09.2020)

Land Matrix (2020). Datos. Por investor country. Recuperado de: https://landmatrix.org/data/by-investor-country/ china/?more $=400($ | 4.09 .2020$)$

Li,Y.y Li-Tao. (20I5). Sugarcane Agriculture and Sugar Industry in China. Sugar Tech, I 7 (I), I-8.

McMichael, P. (2019). Does China's 'going out' strategy prefigure a new food regime?. The Journal of Peasant Studies, 47 (I), I I6-I54.

McMichael, P. (20I2). The land grab and corporate food regime restructuring, The Journal of Peasant Studies, 39 (3-4), $68|-70|$

Mora, S. (2016). Capitalismo, crisis y naturaleza. Un análisis del acaparamiento de tierras dentro y desde el Sur Global. Relaciones Internacionales, (33), 53-73.

Moore, J. (2015b). Cheap Food and Bad Climate: From Surplus Value to Negative Value in the CapitalistWorld-Ecology. Critical Historical Studies, 2 (I).

Moore, J. (2015a). Capitalism in the Web of Life: Ecology and the Accumulation of Capital.Verso.

Moore, J. (20I3) El auge de la ecología-mundo capitalista. Laberinto (38), 9-26.

Moore, J. (20/2). Cheap Food y Bad Money Food, Frontiers, and Financialization in the Rise and Demise of Neoliberalism. Review (Fernand Braudel Center), 33 (2-3), 225-26.

Moore, J. (2010). The End of the Road? Agricultural Revolutions in the Capitalist World-Ecology, I450-20 I0. Journal of Agrarian Change, 10 (3), 389-4I3.

Murmis, M y Murmis, M. (20I2). Land concentration and foreign land ownership in Argentina in the context of global land grabbing. Canadian Journal of Development Studies, 33 (4), 490-508.

Myers, M., y Jie, G. (2016). China's agricultural investment in Latin America: A Critical Assessment. China and Latin American report. The Dialogue.

Shuping, N. (29.05.2020). China Ends 95\% Sugar Tariffs But Keeps Tight Grip on Imports. Bloomberg. Recuperado de: https://www.bloomberg.com/news/articles/2020-05-29/china-ends-95-sugar-tariffs-but-keeps-tight-grip-onimports_(|4.09.2020)

Oliveira, G. de L.T. (20I8). A resistência à apropriação chinesa de terras no Brasil desde 2008. Ideias, 9 (2), 99 -I 32.

Oliveira, G. (2017). Chinese land grabs in Brazil? Sinophobia and foreign investments in Brazilian soybean agribusiness. Globalizations, 15 (I), II4-133.

Oliveira, G. y Schneider, M. (2016). The politics of flexing soybeans: China, Brazil and global agroindustrial restructuring. The Journal of Peasant Studies, 43 (I), 167-194. 
Panitch, L., y Gindin, S. (2013). The Integration of China into Global Capitalism. International Critical Thought, 3 (2), $146-158$.

Peng, S., Tang Q. y Zou Y. (2009). Current Status and Challenges of Rice Production in China. Plant Production Science, $12(1), 3-8$.

Phillips, N. (2009). Coping with China. In Cooper, A. and Heine, J. (Eds.). Which Way Latin America? Hemispheric Politics meets Globalization (pp. 100-12I). United Nations University Press.

PSUV (06.03.20II). Empresa mixta China-Venezuela afianza la soberanía alimentaria. Recuperado de: https:// farmlandgrab.org/l 8293 (I3.09.2020).

Puyana, A. y Costantino, A. (2015). Chinese Land Grabbing in Argentina and Colombia. Latin American Perspectives, 42 (6), $105-119$.

Saguier, M. (2019). Narrativas visuales de la crisis ecológica global. En Kozel, A., Berger, M. y Llobet, V. (eds). El futuro: miradas desde las humanidades (pp. 202-217). UNSAM Edita.

Saguier, M. y Ghiotto, L. (2018). Las empresas transnacionales: un punto de encuentro para la Economía Política Internacional de América Latina. Desafios, 30 (2), I59-190.

Schneider, M. (2017). Dragon Head Enterprises and the State of Agribusiness in China. Journal of Agrarian Change, 17 (I), 3-2I

Thomas, N. (20I3). Going out: China's food security from Southeast Asia. The Pacific Review, 26 (5), 53 I-562.

USDA (United States Department of Agriculture). (2019). China's Peoples Republic of Oilseeds and Products Annual. Foreign Agricultural Center.

White, B., Borras S., Hall, R., Scoones, I.,Wolford,W. (20I2). "The new enclosures: critical perspectives on corporate land deals". The Journal of Peasant Studies, 39 (3-4). 619-647.

Woods, K, y Kramer, T. (20I2). Financing Dispossession - China's Opium Substitution Programme in Northern Burma. Transnational Institute (TNI) Report.

Xu, P., Su, H. y Lone, T. (2018). Chinese consumers' willingness to pay for rice. Journal of Agribusiness in Developing and Emerging Economies, 8 (2), 256-269.

Zhan, S., Zhang, H y He, D. (2018). China's flexible overseas food strategy: food trade and agricultural investment between Southeast Asia and China in 1990-20I5. Globalizations, I5 (5), 702-72I.

Zhang, H. (2019). When Food Crosses Borders: Paradigm Shifts in China's Food Sectors and Implications forVietnam. In Ehlert, J. y Faltmann, N (Eds.). Food Anxiety in a Globalizing Vietnam (pp. 267-299). Palgrave Macmillan.

Zhang, H. y Cheng, G. (20I6). China's Food Security Strategy Reform: An emerging global agricultural policy. In Wu, F. y Zhang, H. (Eds). China's Global Quest for Resources. Energy, Food and Water (pp. 23-4I). Routledge.

Zhang, J. (2019). Beyond the 'Hidden Agricultural Revolution' and 'China's Overseas Land Investment': Main Trends in China's Agriculture and Food Sector. Journal of Contemporary China, 28 (I I9), 746-762.

Zhou, J (2017). Chinese agrarian capitalism in the Russian Far East. Third World Thematics, I (5), 6I2-632. 


\section{RELACIONES INTERNACIONALES}

Revista académica cuatrimestral de publicación electrónica

Grupo de Estudios de Relaciones Internacionales (GERI)

Universidad Autónoma de Madrid, España

https://revistas.uam.es/relacionesinternacionales

ISSN 1699 - 3950

f facebook.com/RelacionesInternacionales

3. twitter.com/RRInternacional 